\title{
Aging pet cats develop neuropathology similar to human Alzheimer's disease
}

\author{
Jenna Klug ${ }^{\mathrm{a}}$, Jessica M. Snyder ${ }^{\mathrm{a}}$, Martin Darvas ${ }^{\mathrm{b}}$, Denise M. Imai ${ }^{\mathrm{c}}$, Molly Church $^{\mathrm{d}}$, Caitlin Latimer ${ }^{\mathrm{b}}$, \\ C. Dirk Keene ${ }^{\mathrm{b}}$, Warren Ladiges ${ }^{\mathrm{a}, *}$ \\ ${ }^{a}$ Department of Comparative Medicine, School of Medicine, University of Washington, Seattle, WA, USA. \\ ${ }^{b}$ Division of Neuropathology, Department of Laboratory Medicine and Pathology, School of Medicine, University of \\ Washington, Seattle, WA, USA. \\ ${ }^{c}$ Comparative Pathology Labortatory, School of Veterinary Medicine, University of California, Davis, CA, USA. \\ ${ }^{d}$ Department of Pathobiology, School of Veterinary Medicine, University of Pennsylvania, Philadelphia, PA, USA.
}

\begin{abstract}
Aging pet cats can spontaneously develop $A \beta$ deposition and tauopathy (including neurofibrillary tangle formation) with neuronal loss in a similar distribution and with similar characteristics to Alzheimer's disease (AD) in humans. These three major pathologies that characterize AD rarely occur spontaneously in other nonhuman animals. In addition, cats develop cognitive impairment with increasing age, and some studies show an association with neuronal lesions. These features suggest that the aging pet cat may be a more reliable spontaneously occurring model to investigate pathogenesis of, and therapeutic interventions for, AD compared to other domestic animals such as pet dogs. This review describes the unique translational potential of the domestic cat as a natural model of $\mathrm{AD}$, with reference to other animal models of $\mathrm{AD}$.
\end{abstract}

Keywords: Alzheimer's disease, pet cats, feline neuropathology, aging, feline cognitive dysfunction syndrome

\section{Introduction}

Alzheimer's disease (AD) is the most common neurodegenerative disease in humans, accounting for nearly two thirds of dementia cases and affecting roughly 35 million people worldwide [1]. The disease is characterized by pathologic accumulations of two types of protein aggregates in specific brain regions, which include plaques of amyloid beta $(\mathrm{A} \beta)$ peptide in the neuropil, and neurofibrillary tangles (NFTs) composed of hyperphosphorylated tau (pTau) protein found both within neurons and within the neuropil as ghost tangles [2-3], but generally both of these accumulations are thought to be necessary for progressive neuronal dysfunction, neuronal loss and cognitive dysfunction in AD. The focus of pharmacologic trials has shifted towards treating individuals in early stages of disease, with the goal to slow or prevent disease progression

\footnotetext{
* Corresponding author: Warren Ladiges

Mailing address: Department of Comparative Medicine, School of Medicine, University of Washington, Seattle, WA, USA.

Email: wladiges@uw.edu
}

Received: 07 September 2020 / Accepted: 11 September 2020 and limit disability [3-4]. Genetically engineered rodent models have been useful to identify candidate drugs for early preclinical studies, however, candidate drugs validated in these models have generally been unsuccessful in clinical trials [5]. Challenges translating therapies from mice to humans may be explained by inherent differences in neuroanatomy, lifespan, and physiology between the species, as well as the fact that most mouse models result from manipulation of one or more specific genes implicated in less common familial forms of the disease rather than sporadic AD. Understanding the mechanisms and pathogenesis in an animal model that naturally develops neuropathologic lesions similar to AD is almost certainly necessary for development of effective translational therapeutic strategies targeting early stages of the disease.

Several animal species have been shown to develop one or more age-related lesions that are comparable to AD. Ideally, animal models should possess 2 histopathologic hallmarks of $\mathrm{AD}$ : $\mathrm{A} \beta$ peptide deposition (plaques) and tau pathology (NFTs) [1]. In general, nonhuman primates (NHPs) and dogs develop spontaneous $\mathrm{A} \beta$ deposition with age, but do not reliably recapitulate tau pathology [6-7]. Wild-type rodents do not spontaneously form plaques or NFTs, but genetically engineered mice have been developed that have single, double or multiple mutations in genes responsible for the production of the $A \beta$ and/or 
tau proteins. Plaques are formed in these models by overexpression of mutant human amyloid precursor protein (APP), resulting in overexpression of total $A \beta$ beyond physiologic levels or increasing the proportion of $A \beta_{1-42}$, which is more prone to pathogenic aggregation and has a higher neurotoxicity profile comparted to $A \beta_{1-40}$. NFTs occur in some mouse models expressing mutations of microtubule associated protein tau (MAPT), which causefrontotemporal dementia, not $\mathrm{AD}$, in people. Although helpful in preclinical trials, candidate drugs tested in rodent transgenic models have very poor performance in clinical trials [5]. By contrast, a limited number of studies have shown that domestic cats can spontaneously develop both histopathologic hallmarks of AD (A $\beta$ deposition and NFTs), as well as associated neuronal loss, in a pattern of distribution similar to humans that progresses with age [56] (Table 1). Additional studies involving large cohorts are needed to further characterize neuropathologic similarities between pet cats and humans and to correlate neuronal lesions with cognitive dysfunction.

\section{$A \beta$ plaques}

A $\beta$ plaques are derived from amyloid precursor protein (APP), which is an integral membrane protein made by neurons and other brain cells, coded by the APP gene [3]. When APP is processed, it is cleaved at an extracellular domain followed by an intracellular domain, and follows one of two pathways. The non-amyloidogenic pathway involves cleavage of the extracellular domain of APP by an $\alpha$-secretase, followed by intramembrous -secretase cleavage, which prohibits the production of $\mathrm{A} \beta$ peptide. Conversely, the amyloidogenic pathway involves cleavage of the extracellular domain of APP followed by intramembranous $\gamma$-secretase, which produces neurotoxic $\mathrm{A} \beta$ peptides. The $\mathrm{C}$ terminus of $\mathrm{A}$ peptide is variable, ranging from 36-43 amino acids in length, based on alterations in the location of cleavage by $\gamma$-secretase [2]. In general, the two dominant forms of $A \beta$ in $A D$ are $A \beta_{1-40}$ and $A \beta_{1-42}$. $\mathrm{A} \beta_{1-42}$ is less soluble, more aggregation prone, and more pathogenic than $A \beta_{1-40}$ [1]. Once $A \beta$ is produced, it accumulates in the extracellular spaces of the brain (parenchymal deposits) and may also be seen in vascular walls (cerebral amyloid angiopathy, CAA). CAA can be seen with, but is not exclusive to AD.

Parenchymal $A \beta$ deposits may be classified into subtypes based on morphology, fibrils (B-sheet conformation), and the surrounding elements (degenerative neurites [i.e. dystrophic neurites] and reactive astrocytes and microglia) [8]. In general, there are two main subtypes: diffuse and focal deposits [1-2]. The focal deposits are composed of a focal spherical dense core of fibrillar $A \beta\left(A \beta_{40}\right.$ and $A \beta_{1-}$ ${ }_{42}$ ) that stains positive for Congo red and thioflavin S. Focal deposits that contain few reactive glia and dystrophic neurites (and thus lack a layered structure) are referred to

Table 1. Summary of relevant publications describing $\mathbf{A} \boldsymbol{\beta}$ and phosphorylated tau (pTau) neuropathology in aged cats. Abbreviations: $4 \mathrm{G} 8$ and 6E10- antibodies for A $\beta$; AT8 and AT100- antibodies for pTau; NFT- neurofibrillary tangles.

\begin{tabular}{|c|c|c|c|c|c|c|c|c|c|c|}
\hline \multirow[b]{2}{*}{ Study } & \multicolumn{6}{|c|}{$\mathbf{A \beta}$} & \multicolumn{4}{|c|}{ Tau } \\
\hline & Age & $\begin{array}{l}\text { Preva- } \\
\text { lence }\end{array}$ & Distribution of plaques & Type of plaques & $\begin{array}{l}\text { Distribution of poorly } \\
\text { circumscribed deposits }\end{array}$ & Type & Age & $\begin{array}{l}\text { Preva- } \\
\text { lence }\end{array}$ & Distribution & Type \\
\hline $\begin{array}{l}\text { Nakamura } \\
\text { et al., } 1996\end{array}$ & $>18 y$ & $3 / 3$ & $\begin{array}{l}\text { cerebral cortex (temporal } \\
\text { lobe }>\text { occipital lobe) }\end{array}$ & $\mathrm{A} \beta_{1-40}$ & cortical neuropil & $\mathrm{A} \beta_{1-40}$ & $\mathrm{n} / \mathrm{a}$ & $\mathrm{n} / \mathrm{a}$ & $\mathrm{n} / \mathrm{a}$ & $\mathrm{n} / \mathrm{a}$ \\
\hline $\begin{array}{l}\text { Brellou } \\
\text { et al., } 2005\end{array}$ & $>17 y$ & $4 / 4$ & $\begin{array}{l}\text { cortical layers of parietal } \\
\text { lobe }\end{array}$ & $\begin{array}{l}\text { strong } A \beta_{1-42+}, \\
\text { weaker } A \beta_{1-40} \\
A \beta_{8-17}\end{array}$ & $\begin{array}{l}\text { cortical layers of the } \\
\text { frontal and parietal } \\
\text { lobes }\end{array}$ & $\begin{array}{l}\text { strong } A \beta_{1-42+} \text {, } \\
\text { weaker } A \beta_{1-40} \\
A \beta_{8-17}\end{array}$ & $\mathrm{n} / \mathrm{a}$ & $\mathrm{n} / \mathrm{a}$ & $\mathrm{n} / \mathrm{a}$ & $\mathrm{n} / \mathrm{a}$ \\
\hline $\begin{array}{l}\text { Head } \\
\text { et al., } 2005\end{array}$ & $>16 y$ & $4 / 5$ & $\begin{array}{l}\text { prefrontal cortex, } \\
\text { parahippocampal gyrus, } \\
\text { parietal cortex, } \\
\text { occipital cortex }>\text { outer } \\
\text { molecular layer dentate } \\
\text { gyrus }\end{array}$ & $4 \mathrm{G} 8$ and $\mathrm{A} \beta_{1-42}$ & $\begin{array}{l}\text { prefrontal cortex, } \\
\text { parahippocampal } \\
\text { gyrus, parietal cortex, } \\
\text { occipital cortex }\end{array}$ & $\begin{array}{l}4 \mathrm{G} 8 \text { and } \\
\mathrm{A} \beta_{1-42} \\
\text { infrequent } \\
\mathrm{A} \beta_{1-16} \\
(\mathrm{n}=3 / 5)\end{array}$ & $>16 y$ & $5 / 5$ & $\begin{array}{l}\text { hippocampus } \\
\text { CA1, subiculum, } \\
\text { and entorhinal/ } \\
\text { parahimppo- } \\
\text { campal cortex in } \\
\text { layers II and V }\end{array}$ & $\begin{array}{l}\text { AT8+, anti-ubiquitin+ } \\
\text { within dystrophic } \\
\text { neurites }(\mathrm{n}=5 / 5) ; \\
\text { AT8+, PHF1+ within } \\
\text { neurons }(\mathrm{n}=2 / 5)\end{array}$ \\
\hline $\begin{array}{l}\text { Gunn-Moore } \\
\text { et al, } 2006\end{array}$ & $>10 \mathrm{y}$ & $7 / 9$ & $\begin{array}{l}\text { cortical layers (deep) of } \\
\text { anterior }>\text { mid-cerebrum }\end{array}$ & $4 \mathrm{G} 8$ & & & $>11 \mathrm{y}$ & $2 / 9$ & $\begin{array}{l}\text { cerebrum, } \\
\text { medulla, } \\
\text { vestibular nuclei }\end{array}$ & AT8+ neurons \\
\hline $\begin{array}{l}\text { Takeuchi } \\
\text { et al, } 2008\end{array}$ & $>10 \mathrm{y}$ & $6 / 6$ & $\begin{array}{l}\text { cerebral cortex and } \\
\text { hippocampus }\end{array}$ & $\mathrm{A} \beta_{1-42}$ & $\begin{array}{l}\text { cerebral cortex and } \\
\text { hippocampus }\end{array}$ & $\mathrm{A} \beta_{1-42}$ & $\mathrm{n} / \mathrm{a}$ & $\mathrm{n} / \mathrm{a}$ & $\mathrm{n} / \mathrm{a}$ & $\mathrm{n} / \mathrm{a}$ \\
\hline $\begin{array}{l}\text { Chambers } \\
\text { et al., } 2015\end{array}$ & $>8 \mathrm{y}$ & $14 / 15$ & cerebral cortex & $\mathrm{A} \beta_{1-42}$ & $\begin{array}{l}\text { intracellular oligomers } \\
\text { in hippocampus }\end{array}$ & $\mathrm{A} \beta_{1-42}$ & $>14 y$ & $9 / 14$ & $\begin{array}{l}\text { entorhinal cortex, } \\
\text { hippocampus }> \\
\text { cerebellar cortex, } \\
\text { locus coeruleus }\end{array}$ & AT8 and AT100 \\
\hline $\begin{array}{l}\text { Poncelot } \\
2019\end{array}$ & $>16 y$ & $9 / 11$ & $\begin{array}{l}\text { temporal and } \\
\text { frontalcortex }\end{array}$ & $\mathrm{A} \beta_{1-42+}$ & $\begin{array}{l}\text { intracellular in } \\
\text { hippocampus }(\mathrm{n}=1 / 9)\end{array}$ & $\begin{array}{l}\mathrm{A} \beta_{1-42} \text { and } \\
\mathrm{A} \beta_{1-40}\end{array}$ & $>18 \mathrm{y}$ & $4 / 6$ & $\begin{array}{l}\text { hippocampus and } \\
\text { cortical subcorti- } \\
\text { cal structures }\end{array}$ & AT8 and PHF1 \\
\hline $\begin{array}{l}\text { Fiock } \\
\text { et al., } 2020\end{array}$ & $>14 y$ & $27 / 28$ & $\begin{array}{l}\text { Cortical layers IV and } \\
\text { VI most prominent }+/- \\
\text { hippocampus and basal } \\
\text { ganglia }\end{array}$ & $6 \mathrm{E} 10+$ & & & $>14 y$ & $4 / 28$ & $\begin{array}{l}\text { entorhinal cortex } \\
>\text { hippocampus, } \\
\text { and neocortex }\end{array}$ & AT8+ \\
\hline
\end{tabular}


as primitive plaques. At a later stage, when they are centrally surrounded by more extensive dystrophic neurons and reactive glia, they are referred to as mature or neuritic plaques (NPs). The diffuse form of A deposits (DP) make up the diffuse or amorphous plaque (DP), which are predominantly $A \beta_{1-42}$. Morphologically they are large (>50 um), poorly limited, stain negative for Congo red and Thioflavin S, are not surrounded by dystrophic neurites, and typically do not have a surrounding glial reaction. While both DPs and NPs can be found in normal aging, the degree of cognitive impairment in AD patients is correlated with the severity of NPs, but not DPs. In addition to routine $H \& E$ and special stains, $A \beta$ deposits can also be detected by IHC using antibodies such as $A \beta_{1-16}(6 \mathrm{E} 10)$, $\mathrm{A} \beta_{8-17}(6 \mathrm{~F} 3 \mathrm{D})$, and $A \beta_{17-24}$ (4G8).

Topographically, parenchymal $\mathrm{A} \beta$ deposits are located almost exclusively in the gray matter. The brain regions involved depend on stage of disease, which can be classified into five successive phases (Thal Phases). The main regions involved by phase include: neocortex in phase I; hippocampus and entorhinal cortex in phase II; neostriatum and diencephalic nuclei in phase III; brainstem nuclei in phase IV; cerebellum and additional brainstem nuclei (pontine nuclei and locus coeruleus) in phase V $[2,5]$. Deposition of plaques typically occurs first as DPs, followed by primitive plaques which progress to NPs.

In cats, $A \beta$ deposition has been widely documented to increase with age, beginning by 7.5 years, and typically by 10 years of age [9-10]. Morphologically these are present predominantly as DPs $\left(\mathrm{A} \beta_{1-42}\right)$, and are negative for Congo Red and thioflavin. They are most commonly found in the cerebral cortex, with extension to the hippocampus and basal ganglia. This is comparable to the distribution of DPs in humans, which are most commonly found in the cerebral cortex, basal ganglia, and cerebellar cortex [3]. Although typical NPs have not been identified in cats, another form of $A \beta$ deposition (intracellular $A \beta$ oligomers) has been found to accumulate within hippocampal pyramidal cells of cats 14 years and older [6]. The intracellular $\mathrm{A} \beta$ oligomers were composed of hexamers and dodecomers, and found in the same brain regions as NFTs with associated neuronal loss, similar to AD patients. Collectively, $A \beta$ deposition in cats is most similar to early forms present during normal aging and $\mathrm{AD}$ in humans.

Several other species have been shown to accumulate A deposition with age including pet dogs and nonhuman primates (NHPs), in comparison to cats and humans (Figure 1). Similar to cats, both dogs and NHPs demonstrate agerelated deposition of $A \beta$ the brain reminiscent of some but not all features of $\mathrm{AD}$. In dogs, the $\mathrm{A} \beta$ peptide is identical in sequence to humans, with deposits mainly as DPs beginning at 8 years of age, composed primarily of $A \beta_{1 \text { - }}$ ${ }_{42}$ with minimal $A \beta_{1-40}$, in similar topographic locations to cats and humans [11-12]. Neuritic plaques of $A \beta_{1-40}$ and $\mathrm{A} \beta_{1-42}$ may occur less commonly, and mature NPs are very rare in dogs [1]. In NHPs, age-related $A \beta$ deposition has been documented in great apes, old world monkeys, new world monkeys, and prosimians, although there is a high degree of variability between groups in the age of onset, distribution, amount, and appearance of lesions. In general, DPs are most common across the groups, although old world monkeys display more NP plaques compared to other animals, beginning around 30 years of age $[4,7]$.

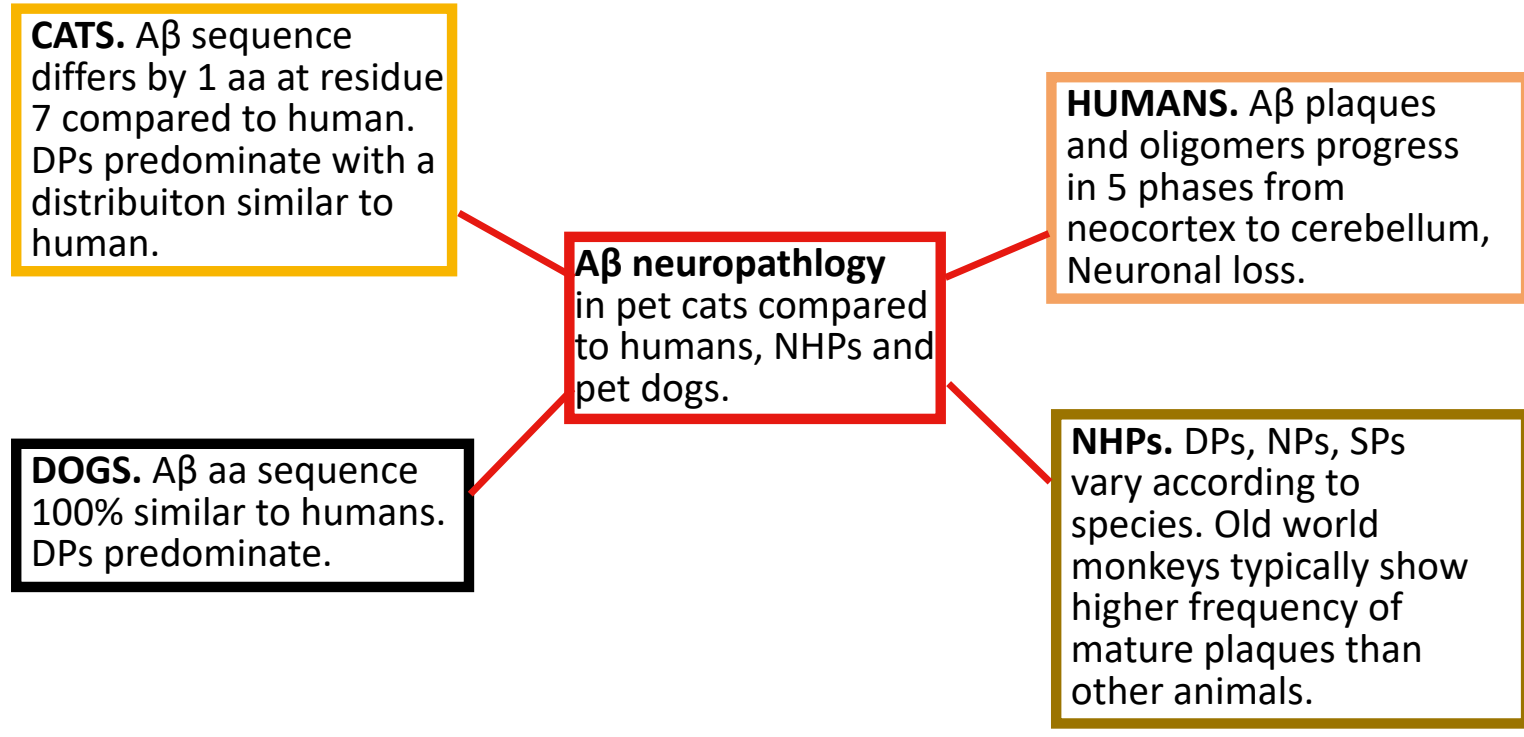

Figure 1. Summary of A $\beta$ neuropathology in pet cats compared to humans, NHPs, and pet dogs. Abbreviations: NHPs- nonhuman primates; DPdendritic plaques; NP- neuritic plaques; SP- senile plaques. 


\section{Tau aggregates (NFTs)}

Tau is a microtubule-binding phosphoprotein in axons that mediates axonal transport, encoded by the MAPT gene [2-3]. Alternative splicing of MAPT yields a total of 6 isoforms in the human adult brain. Specifically, the isoforms differ by the number of amino acids inserted at the $\mathrm{N}$ terminal aspect of the protein $(0,1$ or 2 producing $0 \mathrm{~N}$, $1 \mathrm{~N}$ or $2 \mathrm{~N}$ ) and by the presence of three or four amino acid repeats $(3 R$ and $4 R$ ) in the $C$ terminal part of the protein, which contain the microtubule binding domains [13]. In $\mathrm{AD}$ (a taupathy), tau is shifted from primarily axonal locations to a somatic-dendritic distribution, becomes hyperphosphorylated, ubiquinated, and loses its ability to bind to microtubules, resulting in a misfolded, insoluble tau protein. The specific tau isoforms that accumulate in humans vary across different taupathies. pTau aggregates in $\mathrm{AD}$ consist of hyperphosporylated $3 \mathrm{R}$ and $4 \mathrm{R}$ isoforms [2]. In addition, the active form glycogen synthase kinase-3 (GSK3), a tau protein kinase, has been found in association with tau lesions and at increased expression levels in AD brains [13]. Tau aggregates may be present in the neuron cell body, dendrites, and axons. In the cell body, tau aggregates progress from pretangle lesions (diffuse or granular phospho-tau immunoreactivity in the absence of fibrillary structure) then become fibrillary, forming neurofibrillary tangles (NFTs). NFTs that form in the neuronal cytoplasm result in neuronal degeneration and death, after which the NFTs persist in the neuropil as ghost tangles. Tau aggregates may also accumulate in dendrites, where they are known as neuropil threads. Lastly, tau aggregates can be present in axons, where they are visualized as fine tau-positive processes that make up the neuritic component (crown) of the focal plaque.

Tau aggregates are usually detected by IHC using immunostaining with anti-tau antibodies such as AT8, PHF1, and anti-3R and anti-4R. Special stains such as silver stains and thioflavin $\mathrm{S}$ can also be used [1]. Ultrastructurally, tau aggregates in AD are made of two filaments paired in a helical structure (PHFs). Straight filaments have also been seen, but usually these are more suggestive of other taupathies such as progressive supranuclear palsy (PSP) and Pick's disease (PiD) [13]. In general, the development of $A \beta$ plaques is considered to precede and accentuate the develop of NFTs in human AD [14]. The burden of tau pathology progresses from medial temporal lobe to neocortex describred through Braak stages, as detected by AT8 immunohistochemistry. The stages sequentially involve the medial temporal cortex (stage I), hippocampus CA1 (stage II), subiculum and other regions of hippocampus and medial temporal cortex (stage III and IV) and other areas of neocortex (stage V and VI) [2].

Aging cats have been shown to develop tauopathies that share many features to human AD. For example, adult cats express 6 total tau isoforms, including the hyperphosphorylated $3 \mathrm{R}$ and $4 \mathrm{R}$ isoforms, which can form aggregates in the presence of $\mathrm{A} \beta$ as in human $\mathrm{AD}[6,13]$.
Cats also show increased expression of GSK3 (tau protein kinase) in association with tauopathies and regions of neuronal loss at 15 years of age [15]. Morphologically, neurons with intracellular phosphorylated tau in aging cats have been noted to display a sprouting response similar to human AD [15]. Several forms of tau aggregates reported in cats include: pretangles, threads, dystrophic neurites, NFTs and ghost tangles [16]. Ultrastructurally, cat NFTs are similar to those in humans, consisting of some straight filaments but mostly paired twisted patterns of filaments. These features of feline NFTs accompanied by neuronal loss and occurring in intracellular oligomers in the same brain region (hippocampus), have also been described in transgenic mouse models and human AD patients, and thus represent a potentially high impact naturally occurring model of the disease [5] compared to pet dogs and NHP's (Figure 2).

Although most other aging nonhuman animals do not spontaneously develop NFTs and neuronal loss, abnormal tau phosphorylation has been described in a number of species including sheep and goats, bison, bears, degu, and wolverines $[1,13]$. Dogs have not been shown to develop NFTs, but do show increased phorphorylation of tau that may represent pretangle pathology. In NHPs, NFTs are relatively rare, and when present are usually focal, mild, and not associated with neuronal loss $[4,7]$.

\section{Cognitive dysfunction}

Recent clinical and research criteria have identified three progressive stages of human AD: preclinical, prodromal, and dementia [2]. During the preclinical period, there are no symptoms of cognitive dysfunction. However, neuropathology associated with AD (accumulation of A $\beta$ deposition followed by tau pathology and neuronal degeneration/synaptic dysfunction) occurs during this period and may precede initial symptoms by decades [14]. Clinical signs of mild cognitive impairment occur during the prodromal stage followed by progression to dementia (clinical diagnosis of AD) based on cognitive-behavioral status. Typically, AD presents as an initial decline in episodic memory, followed by progressive decline across multiple cognitive functions or intellectual symptoms (ex: amnesia, aphasia, apraxia, agnosia) and behavioral changes (personality changes, depression, hallucinations, delusions) that impair social function and difficulties with daily living. Therefore, clinical AD is considered a late stage of disease progression, and biomarkers characterizing preclinical and prodromal stages are essential for interventions aimed at preventing or reversing disease progression.

The association between age-related neurodegenerative lesions and cognitive dysfunction in cats is not as well described as for humans or dogs. However, both dogs and cats can develop age-related deterioration in cognitive functions manifested as behavioral changes that are similar to cognitive domains affected in AD [14]. Cognitive dysfunction syndrome (CDS) is the term used to describe 


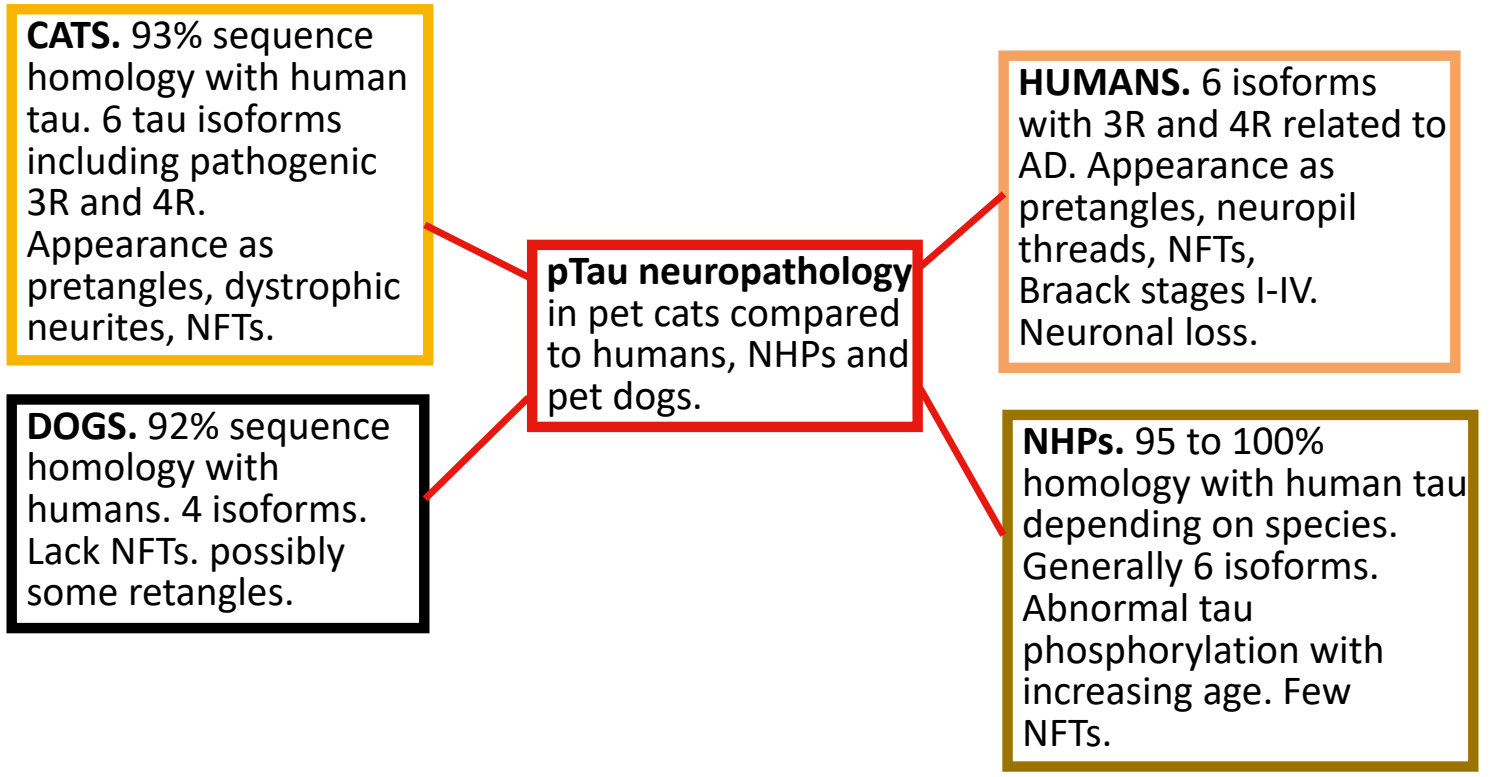

Figure 2. Summary of pTau neuropathology in pet cats compared to humans, NHPs, and pet dogs. Abbreviations: NHPs- nonhuman primates; NFT- neurofibrillary tangles.

cognitive decline in geriatric cats and dogs after excluding underlying causes of illness or behavior effects of drugs. CDS is estimated to affect roughly one third ( 28 percent) of cats 11-14 years old, and 50 percent of cats 15 years of age or older [19]. The incidence of CDS is similar in clinical settings in dogs, which is estimated to affect roughly 28 percent of dogs 11-12 years old, and 68 percent of dogs over 15 years old (although lifespan and biological age in dogs varies by breed) [9, 17]. Human dementia displays similar trends, affecting roughly $1-3$ percent of people 65 70 years old and 50 percent of people over 85 years of age. In cats, there is not yet a validated relationship between cognitive/behavioral dysfunction and extent of $\mathrm{A} \beta$ deposition [15], but aged cats that display signs of behavioral dysfunction have been found to have $A \beta$ plaques $[16$, 18]. An association between tau pathology and CDS also remains to be more clearly clarified, but several studies in aged cats showed that all cats affected with tau pathology ( $4 / 4$ cats $>15$ y and $5 / 5$ cats $16-21$ y) displayed changes in behavior suggestive of cognitive/memory impairment $[11,13]$. The pattern of $\mathrm{A} \beta$ deposition in dogs has been documented to increase with age, and extent of deposition is positively correlated with cognitive impairment [910]. Although dogs do not develop NFTs as in humans with $\mathrm{AD}$, a study of multiple breeds of dogs demonstrated that intracellular phosphorylated tau (Ser396) expression in neurons and astrocytes of the cerebral cortex and hippocamus was associated with cognitive dysfunction. Agerelated deficits in memory and attention, deposits of amyloid plaques, and atrophy and/or loss of cholinergic and monoaminergic neurons are well documented in NHP [4]. Further studies of larger cohorts are needed to expand on the neuropathologic and cognitive changes that have been characterized thus far in aging cats. Studies could be designed that borrow from canine studies in which samples are obtained from clinical settings, while also addressing issues identified in feline studies including behavioral changes relevant or more common in feline CDS compared to dogs. For example, archived brain samples from potentially large cohorts could be accessed from veterinary clinics retrospectively from young and old cats that were euthanized or died spontaneously from various disorders, excluding those with major brain lesions such as neoplasia or inflammatory disease evident grossly or microscopically. Corresponding clinical records could be subjected to a scoring system to group cats into cohorts of cognitive decline (present/absent) or adapted/modified from one of the several available scoring systems for screening severity of age-related cognitive disorders in pet cats (ex: absent, mild, moderate severe). Available clinical and historical data to evaluate for the presence of concurrent disease would include physical exam findings, laboratory test results including bloodwork and urinalysis, results of screening for infectious disease such as FeLV and FIV, imaging and medications [14-16]. Examples of common diseases that may impact behavior in cats (and people) include hyperthyroidism, chronic renal disease, hypertension, osteoarthritis and diabetes mellitus [17, 19]. Ideally, with a large enough cohort, these diseases could be randomized between groups of cats with cognitive dysfunction and those without. Collectively, studies that incorporate these elements could help to better address the question of whether neuropathologic lesions of AD correlate with clinical signs of behavioral/cognitive dysfunction, and whether there are any effects of comorbidies and breed on the extent of neurolopathologic changes. 


\section{Conclusion}

In summary, A deposition and tauopathy (including NFT formation) with neuronal loss can spontaneously develop in aging pet cats in a similar distribution and with similar characteristics to human AD. Other nonhuman mammalian animals rarely develop all three of the major pathologies that characterize AD. Concurrent with the presence of neuropathologic lesions, cats have been reported to develop cognitive impairment with increasing age; however, the correlation with neuronal lesions is not yet fully validated in this species. The presence of AD-like neuropathology positions the aging pet cat as a potentially high impact model to investigate pathogenesis and therapeutic interventions for $\mathrm{AD}$ with potentially superior translational and preclinical predictive power compared to pet dogs and nonhuman primates. However, additional research on large cohorts of pet cats is needed to confirm these exciting and novel but preliminary observations.

\section{Declarations}

Authors' contributions: Jenna Klug wrote the first draft. All co-authors contributed to editing and additions. Warren Ladiges did the final editing.

Conflicts of interest: The authors declare no conflicts of interest.

Consent for publication: All authors consent to the publication of this manuscript.

\section{References}

1. Youssef S A, Capucchio M T, Rofina J E, et al. Pathology of the aging brain in domestic and laboratory animals, and animal models of human neurodegenerative diseases. Veterinary pathology, 2016, 53(2): 327-348.

2. Calderon-Garcidueñas A L, Duyckaerts C. Alzheimer disease. Handbook of clinical neurology. Elsevier, 2018, 145: 325-337.

3. Kumar V, Abbas A K, Fausto N, et al. Robbins and Cotran pathologic basis of disease, professional edition e-book. Elsevier health sciences, 2014.

4. Emborg M E. Nonhuman primate models of neurodegenerative disorders. ILAR journal, 2017, 58(2): 190-201.

5. Fiock K L, Smith J D, Crary J F, et al. $\beta$-amyloid and tau pathology in the aging feline brain. Journal of Comparative Neurology, 2020, 528(1): 112-117.

6. Chambers J K, Tokuda T, Uchida K, et al. The domestic cat as a natural animal model of Alzheimer's disease. Acta neuropathologica communications, 2015, 3(1): 78.

7. Heuer E, F Rosen R, Cintron A, et al. Nonhuman primate models of Alzheimer-like cerebral proteopathy. Current pharmaceutical design, 2012, 18(8): 1159-1169.

8. Brellou G, Vlemmas I, Lekkas S, et al. Immunohistochemical investigation of amyloid $ß$-protein $(A ß)$ in the brain of aged cats. Histology and histopathology, 2005.

9. Vite $\mathrm{C} \mathrm{H}$, Head E. Aging in the canine and feline brain. Veterinary Clinics: Small Animal Practice, 2014, 44(6): 1113-1129.

10. Yu C H, Song G S, Yhee J Y, et al. Histopathological and immunohistochemical comparison of the brain of human patients with Alzheimer's disease and the brain of aged dogs with cognitive dysfunction. Journal of comparative pathology, 2011, 145(1): 45-58.

11. Chambers J K, Mutsuga M, Uchida K, et al. Characterization of $A \beta p N 3$ deposition in the brains of dogs of various ages and other animal species. Amyloid, 2011, 18(2): 63-71.

12. Takeuchi Y, Uetsuka K, Murayama M, et al. Complementary distributions of amyloid- $\beta$ and neprilysin in the brains of dogs and cats. Veterinary pathology, 2008, 45(4): 455466.

13. Poncelet L, Ando K, Vergara C, et al. A 4R tauopathy develops without amyloid deposits in aged cat brains. Neurobiology of aging, 2019, 81: 200-212.

14. M, Nichol J, Araujo J A. Cognitive dysfunction syndrome: a disease of canine and feline brain aging. Veterinary Clinics: Small Animal Practice, 2012, 42(4): 749-768.

15. Head E, Moffat K, Das P, et al. $\beta$-Amyloid deposition and tau phosphorylation in clinically characterized aged cats. Neurobiology of aging, 2005, 26(5): 749-763.

16. Gunn-Moore D A, McVee J, Bradshaw J M, et al. Ageing changes in cat brains demonstrated by $\beta$-amyloid and AT8-immunoreactive phosphorylated tau deposits. Journal of feline medicine and surgery, 2006, 8(4): 234-242.

17. Gunn-Moore D, Moffat K, Christie L A, et al. Cognitive dysfunction and the neurobiology of ageing in cats. Journal of Small Animal Practice, 2007, 48(10): 546-553.

18. Nakamura S, Nakayama H, Kiatipattanasakul W, et al. Senile plaques in very aged cats. Acta neuropathologica, 1996, 91(4): 437-439.

19. Gunn-Moore D A. Cognitive dysfunction in cats: clinical assessment and management. Topics in companion animal medicine, 2011, 26(1): 17-24.

Cite this article as: Klug J, Snyder J M, Darvas M, et al. Aging pet cats develop neuropathology similar to human Alzheimer's disease[J]. Aging Pathobiology and Therapeutics, 2020, 2(3): 120-125. 